\title{
Verwertbarkeit privater Dashcam-Aufzeichnungen im Strafverfahren
}

\author{
Matthias Maager*
}

Trotz der steigenden Zahl an privaten Dashcams, welche auf schweizerischen Strassen eingesetzt werden, bestehen erhebliche Rechtsunsicherheiten darüber, ob Dashcam-Aufzeichnungen im Strafverfahren verwertet werden dürfen. Der Autor widmet sich deshalb der Frage, unter welchen Voraussetzungen zivil- und datenschutzrechtswidrige Dashcam-Aufzeichnungen, die durch Private erhoben wurden, im Strafverfahren verwertbar sind. Es zeigt sich, dass solche Aufzeichnungen verwertet werden dürfen, wenn sie von den Strafverfolgungsbehörden rechtmässig hätten erlangt werden können und eine Interessenabwägung für deren Verwertung spricht. Ferner sind rechtswidrige Dashcam-Aufzeichnungen zugunsten des Beschuldigten zuzulassen, wenn sie diesen entlasten.

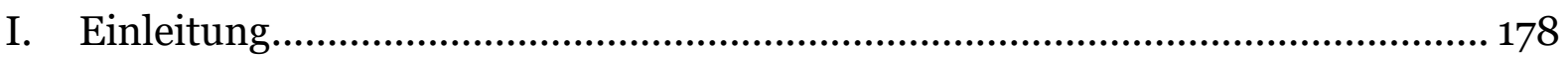

II. Zulässigkeit von privaten Dashcam-Aufzeichnungen.................................. 178

1. Zivilrechtliche Betrachtung ............................................................... 178

2. Datenschutzrechtliche Betrachtung................................................... 179

3. Strafrechtliche Betrachtung ............................................................... 182

III. Verwertbarkeit von Videoaufnahmen durch die Strafverfolgungsbehörden ... 182

IV. Verwertbarkeit privat erhobener Dashcam-Aufzeichnungen............................184

1. Beweisverwertungsverbote und privat beschaffte Beweise ......................... 184

2. Zivil- und datenschutzrechtswidrig erhobene Dashcam-Aufzeichnungen......... 185

3. (Straf-)rechtswidrig erlangte Dashcam-Aufzeichnungen ............................ 188

4. Rechtmässige bzw. gerechtfertigte Dashcam-Aufzeichnungen..................... 195

5. Entlastende Dashcam-Aufzeichnungen als Sonderfall .................................... 195

6. Prüfschema zur Verwertbarkeit privater Dashcam-Aufzeichnungen ............ 197

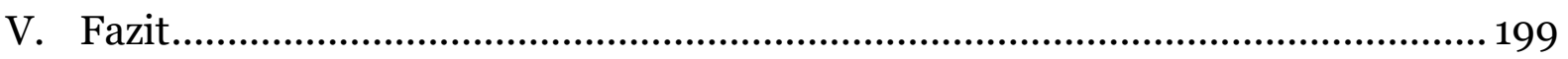

Zitiervorschlag: Matthias Maager, Verwertbarkeit privater Dashcam-Aufzeichnungen im Strafverfahren, in: sui-generis 2018, S. 177

URL: $\quad$ sui-generis.ch/67

DOI: $\quad$ https://doi.org/10.21257/sg.67

* Matthias Maager (matthias.maager[at]bluewin.ch), MLaw, schloss sein Studium an der Universität Zürich im Frühling 2018 ab und ist juristischer Mitarbeiter in einer Anwaltskanzlei in Wil SG. Im Anschluss absolviert er ein Praktikum am Bezirksgericht Weinfelden zur Vorbereitung auf die Anwaltsprüfung. Dieser Beitrag ist eine überarbeitete und gekürzte Fassung seiner Masterarbeit. 


\section{Einleitung}

1 Rund 5,77 Milliarden Schweizer Franken Unfallkosten wurden im Jahr 2014 allein durch den privaten motorisierten Personenverkehr verursacht. ${ }^{1}$ Häufig steht diesen hohen Kosten eine unbefriedigende Beweislage bei Verkehrsunfällen gegenüber. Nicht zuletzt deshalb erfreuen sich in der Schweiz sogenannte Dashcams, welche man bisweilen vor allem aus dem Ausland kennt, wachsender Beliebtheit.

Unter dem gesetzlich nicht definierten Begriff der Dashcam versteht man im Allgemeinen kleine, auf dem Armaturenbrett (engl. «dashboard») oder anderweitig im Motorfahrzeug angebrachte Kameras, welche den Strassenverkehr aufzeichnen. ${ }^{2}$ In Bezug auf die Funktionsweise bestehen zwei grundlegende Aufnahmemodi. Zum einen kann eine Daueraufnahme vorgenommen werden, welche während der gesamten Fahrt aufzeichnet und alles abspeichert. Zum anderen ist ein sog. Schleifenmodus (auch Ringspeicher genannt) möglich, welcher die getätigten Aufzeichnungen nach einer bestimmten Zeit durch Neuaufnahmen überspielt. Eine nachhaltige Speicherung der Abschnitte kann in diesem Fall (manuell) durch Betätigung eines Notfallknopfes oder (automatisch) durch eine sog. Schocksteuerung bzw. einen Unfallsensor erfolgen. 3

\footnotetext{
Bundesamt für Statistik, Kosten und Finanzierung des Verkehrs: Unfall-, Umwelt- und Gesundheitskosten vom 26. September 2017.

2 Starnecker Tobias, Videoüberwachung zur Risikovorsorge: Body-Cam zur Eigensicherung und Dashcam zur Beweissicherung; eine verfassungs- und datenschutzrechtliche Analyse, Berlin 2017, S. 257.

3 Bachmeier Werner, Dash-Cam \& Co. - Beweismittel der ZPO?, in: DAR 1/2014, S. 16; Starnecker (Fn. 2), S. 260.
}

3 Trotz der zunehmenden Zahl an Dashcams, welche auf Schweizer Strassen eingesetzt werden, bestehen erhebliche Rechtsunsicherheiten darüber, ob bzw. inwieweit solche von Privaten erhobenen Aufzeichnungen im Strafverfahren verwertet werden dürfen. Anfangs 2017 lagen - soweit ersichtlich - noch keine ober- bzw. bundesgerichtliche Entscheide diesbezüglich vor. Dies hat sich unterdessen durch die Entscheide des Kantonsgerichts Schwyz 4 sowie des Obergerichts des Kantons Zug5 geändert. Zudem musste sich das Bundesgericht erstmals explizit mit der Verwertbarkeit von Dashcam-Aufzeichnungen befassen. ${ }^{6}$

\section{Zulässigkeit von privaten Dashcam-Aufzeichnungen}

\section{Zivilrechtliche Betrachtung}

4 Zivilrechtlich müssen Dashcam-Aufzeichnungen primär Art. 28 ZGB (privatrechtlicher Schutz vor Persönlichkeitsverletzungen)7 standhalten. Gemäss Art. 28 Abs. 1 ZGB kann derjenige, der widerrechtlich in seiner Persönlichkeit verletzt wird gegen jeden, der an der Verletzung mitwirkt, das Gericht anrufen.

5 In den Schutzbereich des Persönlichkeitsrechts fällt insbesondere das Recht am eigenen Bild als Teilgehalt des Rechts auf informationelle Selbstbestimmung. ${ }^{8}$ Es besagt, dass prinzipiell niemand ohne

$4 \overline{\text { Entscheid des Kantonsgerichts Schwyz STK } 2017}$ 1 vom 20. Juni 2017.

5 Entscheid des Obergerichts des Kantons Zug S 201657 vom 11. Mai 2017; siehe hinten Fn. 36.

6 Urteil des Bundesgerichts 6B_758/2017 vom 26. September 2017; siehe hinten Fn. 36.

7 BGE 136 III 410 E. 2.2 S. 412.

8 Brückner Christian, Das Personenrecht des ZGB: (ohne Beurkundung des Personenstandes), Zürich 2000, Rz. 627. 
seine Zustimmung abgebildet werden darf.9 Zwar stellt nicht jede Aufnahme einer Person im öffentlich zugänglichen Raum bereits eine Persönlichkeitsverletzung dar, ${ }^{10}$ etwa wenn jemand bloss Teil der auf einer Fotografie sichtbaren Landschaft, der Umgebung oder eines Ereignisses ist (sog. Beiwerk). ${ }^{11}$ Allerdings ist bei Personenaufnahmen auf öffentlichen Strassen und Plätzen nur mit Zurückhaltung davon auszugehen, dass jemand blosses Beiwerk darstellt (insbesondere bei weniger belebten Gegenden, aber auch bei zentralen innerstädtischen Plätzen und Strassen, sofern einzelne Personen gut sichtbar sind). ${ }^{12}$ Die Videoaufzeichnung im öffentlich zugänglichen Raum stellt damit regelmässig eine Verletzung des Rechts am eigenen Bild und damit eine Persönlichkeitsverletzung im Sinne von Art. 28 Abs. 1 ZGB dar. ${ }^{13}$

6 Nicht widerrechtlich ist eine Persönlichkeitsverletzung dann, wenn sie durch einen Rechtfertigungsgrund gedeckt ist. ${ }^{14}$ Da der in Kapitel II.2 erwähnte Art. 13 Abs. 1 DSG diesen in Art. 28 Abs. 2 ZGB verankerten Grundsatz übernimmt, wird auf das sogleich folgende Kapitel verwiesen.

9 Meili Andreas, in: Honsell/Vogt/Geiser (Hrsg.), Basler Kommentar, Zivilgesetzbuch I (Art. 1-456 ZGB), 5. Aufl., Basel 2014 (nachfolgend zit.: Meili, in: BSK-ZGB I), Art. 28 Rz. 19.

10 Müller Lucien, Videoüberwachung in öffentlich zugänglichen Räumen - insbesondere zur Verhütung und Ahndung von Straftaten, Diss. Univ. St. Gallen 2011, Zürich/St. Gallen 2011, S. 322.

11 Meili, in: BSK-ZGB I (Fn. 9), Art. 28 Rz. 20.

12 Urteil des Bundesverwaltungsgerichts A-7040/ 2009 vom 30. März 2011, E. 8.2.4; Müller (Fn. 10), S. 322.

13 Müller (Fn. 10), S. 323.

14 Vgl. Art. 28 Abs. 2 ZGB.

\section{Datenschutzrechtliche Betrachtung}

7 Das Aufzeichnen mittels Dashcam stellt eine Datenbearbeitung im Sinne von Art. 2 Abs. 1 DSG dar. Einerseits liegen regelmässig Personendaten vor, da sich etwa mithilfe der Fahrzeugkennzeichen problemlos ein Personenbezug herstellen lässt. ${ }^{15}$ Andererseits stellen Vorgänge wie das Erfassen oder Aufbewahren von (Dashcam-)Bildern eine Bearbeitung von Personendaten im Sinne von Art. 3 lit. e DSG dar.

8 Aus Sicht des Gesetzgebers ist allerdings nicht jede Bearbeitung von Personendaten per se problematisch, sondern lediglich jene, die eine widerrechtliche Persönlichkeitsverletzung verursacht. ${ }^{16}$ Eine unwiderlegbare Vermutung der Persönlichkeitsverletzung gilt insbesondere bei einem Verstoss gegen die Bearbeitungsgrundsätze des Datenschutzgesetzes. ${ }^{17}$ Im Zusammenhang mit dem Filmen mittels Dashcams ist vordergründig ein Verstoss gegen die datenschutzrechtlichen Grundsätze der Erkennbarkeit sowie der Verhältnismässigkeit zu prüfen.

9 Der in Art. 4 Abs. 4 DSG festgehaltene Grundsatz der Erkennbarkeit verlangt im Bereich der Videoüberwachung, dass die betroffenen Personen über die Tatsache und den Zweck der Überwachung in Kenntnis gesetzt werden. Die Videokameras müssen so angebracht sein, dass

15 Vgl. BGE 138 II 346 E. 6.5 S. 356.

16 Wermelinger Amédéo, in: Baeriswyl/Pärli (Hrsg.), Stämpflis Handkommentar zum Datenschutzgesetz (DSG), Bern 2015 (nachfolgend zit.: Wermelinger, in: SHK-DSG), Art. 12 Rz. 1.

17 Rosenthal David, in: Rosenthal/Jöhri, Handkommentar zum Datenschutzgesetz: sowie weiteren, ausgewählten Bestimmungen, Zürich/Basel 2013, Art. 12 Rz. 2 f. 
diese für den Einzelnen offensichtlich erkennbar sind, andernfalls muss deutlich (durch gut sichtbare Hinweisschilder) über den Einsatz der Videoüberwachung informiert werden. ${ }^{18}$ Die Dashcams, welche an der Windschutzscheibe, auf dem Armaturenbrett oder anderweitig im Fahrzeug angebracht werden, zeichnen sich durch ihre geringe Grösse aus und sind damit für betroffene Personen nicht (ohne weiteres) erkennbar. Der datenschutzrechtliche Grundsatz der Erkennbarkeit wird bei der Verwendung von Dashcams damit in der Regel verletzt. ${ }^{19}$

10 Der datenschutzrechtliche Verhältnismässigkeitsgrundsatz ist dann verletzt, wenn der Datenbearbeiter mehr Daten bearbeitet, als er objektiv tatsächlich benötigt bzw. wenn der Eingriff nicht in einem vernünftigen Verhältnis zum vom Bearbeiter verfolgten Interesse steht. Angesprochen sind die Eignung, Erforderlichkeit sowie die Zumutbarkeit der Datenbearbeitung. ${ }^{20}$

Zur Beweissicherung und zur Verhütung von Straftaten kann eine Dashcam-Aufzeichnung durchaus als geeignetes Mittel bezeichnet werden.

18 Maurer-Lambrou Urs/Steiner Andrea, in: Maurer-Lambrou/Blechta (Hrsg.), Basler Kommentar, Datenschutzgesetz/Öffentlichkeitsgesetz, 3. Aufl., Basel 2014 (nachfolgend zit.: BearbeiterIn, in: BSK-DSG), Art. 4 Rz. 38; Mathis Aeppli Barbara, Checkliste Videoüberwachung, in: digma 2003, S. 23.

19 So auch der Eidgenössische Datenschutz- und Öffentlichkeitsbeauftragte (EDÖB) sowie Haag Sophie, Die private Verwendung von Dashcams und der Persönlichkeitsschutz, in: Schaffhauser (Hrsg.), Jahrbuch zum Strassenverkehrsrecht 2016, Bern 2016, S. $173 \mathrm{ff}$.

20 Maurer-Lambrou/Steiner, in: BSK-DSG (Fn. 18), Art. 4 Rz. 9 und 11.
12 Zudem dürfte eine Dashcam-Aufzeichnung zur Beweissicherung erforderlich sein. Zwar wären als mildere Mittel konventionelle Methoden - etwa Zeugenaussagen, Sachverständigengutachten oder polizeiliche Ermittlungen - oder modifizierte Dashcams denkbar. Allerdings weisen sowohl konventionelle Mittel als auch modifizierte Dashcams Defizite gegenüber permanent, ohne Schleifenmodus und anlass-unabhängig während der gesamten Fahrt aufzeichnenden Dashcams auf, sodass sie zur Beweissicherung nicht gleich geeignet sind. ${ }^{21}$

13 Schliesslich hat die Datenbeschaffung zumutbar zu sein. Es sollte ein vernünftiges Verhältnis zwischen dem Bearbeitungszweck und der Beeinträchtigung der Persönlichkeit bestehen. ${ }^{22}$ Die Frage der Zumutbarkeit betrifft grundsätzlich kon-

21 So können etwa Sachverständige den Unfallhergang oftmals nur unergiebig rekonstruieren, wohingegen Zeugenaussagen vielfach subjektiv geprägt sind. Demgegenüber rekonstruieren Unfalldatenspeicher das eigene Verhalten nicht umfassend und das Verhalten der anderen Verkehrsteilnehmer gar nicht. Schliesslich sind bei modifizierten Dashcams (etwa mittels Schleifenmodus, kleinen Speicherkarten, Aufprallknopf oder Verringerung der Bildqualität) durchaus Konstellationen denkbar, in welchen die Aufzeichnungen der letzten 30 oder 60 Sekunden vor dem Unfall nicht ausreichen, um das gesamte Unfallgeschehen zu rekonstruieren. Die einzelnen Ausgestaltungsmöglichkeiten einer Dashcam sind vielmehr im Rahmen der Interessenabwägung bzw. der Zumutbarkeitsprüfung zu berücksichtigen; zum Ganzen Starnecker (Fn. 2), S. 312 ff.; a.M. Haag (Fn. 19), S. 176.

22 Botschaft des Bundesrats zum Bundesgesetz über den Datenschutz (DSG) vom 23. März 1988 (BBl 1988 II 413), S. 450; Bei der Beurteilung der Intensität einer Persönlichkeitsbeeinträchtigung kommt es namentlich darauf an, wie sensibel die Daten sind, wie gross das Verletzungspotenzial ist, in welchen Räumen die Überwachung erfolgt sowie welches Verhalten der Einzelne darin preisgibt (zum Ganzen Müller [Fn. 10], S. 329 ff. m.w.H.). 
krete Einzelfälle und läuft im Ergebnis auf eine gesamthafte Abwägung aller betroffenen öffentlichen und privaten Interessen hinaus. ${ }^{23}$

14 Auf Seite des Gefilmten bestehen etwa schützenswerte Datenschutzinteressen im Recht auf Privatsphäre, im Recht am eigenen Bild sowie darin, in öffentlich zugänglichen Räumen von Privaten nicht beobachtet bzw. aufgezeichnet zu werden. ${ }^{24}$ Auf Seite des Filmenden kommen als schützenswerte Bearbeitungsinteressen etwa Interessen an der Verhinderung von Straftaten oder Interessen der Beweissicherung in Frage. ${ }^{25}$ Dem Einsatz datenschutzfreundlicher Technologien kommt bei der Zumutbarkeitsprüfung zentrale Bedeutung zu. ${ }^{26}$

15 Im Ergebnis dürfte vorab das anlasslose bzw. permanente Aufzeichnen mittels Dashcams eine hohe Eingriffsintensität aufweisen und damit als unzumutbar bzw. unverhältnismässig bezeichnet werden. ${ }^{27}$

Wie bereits angesprochen kann eine Persönlichkeitsverletzung nach Art. 13 DSG gerechtfertigt werden. Art. 13 Abs. 1 DSG nennt - gleich wie Art. 28 Abs. 2 ZGB - als Rechtfertigungsgründe die Einwilligung des Verletzten, ein überwiegendes privates oder öffentliches Interesse sowie das Gesetz. $\mathrm{Zu}$ beachten ist hierbei, dass nach der Rechtsprechung

23 BGE 138 II 346 E. 9.2 S. 362.

24 Vgl. BGE 138 II 346 E. 10.3 S. 364 ; vgl. auch Müller (Fn. 10), S. 330 .

25 Vgl. Urteil des Bundesgerichts 6B_536/2009 vom 12. November 2009, E. 3.7; vgl. auch Müller (Fn. 10), S. 330.

26 Starnecker (Fn. 2), S. 317 f. m.w.H.; Müller (Fn. 10), S. 332.

27 Starnecker (Fn. 2), S. 326; ebenso Haag (Fn. 19), S. $175 \mathrm{ff}$. des Bundesgerichts Verstösse gegen Bearbeitungsgrundsätze des Datenschutzrechts nur mit grosser Zurückhaltung gerechtfertigt werden können. ${ }^{28}$

17 Eine gültige Einwilligung in eine persönlichkeitsverletzende DashcamAufzeichnung dürfte regelmässig «von vornherein ausgeschlossen» 29 sein, weil die Dashcam-Aufzeichnungen in der Regel ohne das Wissen der betroffenen Personen erfolgen. Auch fehlt eine gesetzliche Grundlage für den Einsatz von Dashcams durch Private. Persönlichkeitsbeeinträchtigungen ausschliesslich zu Sicherheitszwecken wegen des Vorliegens öffentlicher Interessen sollten grundsätzlich dem Staat vorbehalten sein. 30

18 Ob schliesslich ein überwiegendes privates Interesse für den Dashcam-Einsatz vorliegt, ist anhand einer einzelfallbezogenen Interessenabwägung zu prüfen. ${ }^{31}$

Zwar ist es richtig, dass mittels Dashcams regelmässig lediglich Verkehrsvorgänge auf öffentlichen Strassen und nur Fahrzeuge, nicht aber deren Insassen, abgebildet werden und somit kein Einblick in die engere Privatsphäre des Lenkers gewährt wird, was tendenziell für ein geringfügige Persönlichkeits-

$2 8 \longdiv { \text { BGE } 1 3 6 \text { II } 5 0 8 \text { E. 5.2.4 S. } 5 2 1 \text { und E. 6.3.1 S. } 5 2 3 . }$

29 Urteil des Bundesverwaltungsgerichts A-7040/ 2009 vom 30. März 2011, E. 10.5.2 zu den Fotoaufnahmen im Fall «Google Street View».

30 Müller (Fn. 10), S. 344; vgl. auch Mäder Stefan, Verwertbarkeit privater Dashcam-Aufzeichnungen im Strafprozess, in: AJP 2018, S. 155 ff., S. 165 m.w.H.

31 Rampini, in: BSK-DSG (Fn. 18), Art. 13 Rz. 20; Sowohl der Datenbearbeiter als auch die betroffene Person können nicht nur private, sondern auch öffentliche Interessen geltend machen (Wermelinger, in: SHK-DSG [Fn. 16], Art. 13 Rz. 9). 
verletzung spricht. ${ }^{2}$ Allerdings ist zu beachten, dass solche Videoaufzeichnungen häufig heimlich erfolgen.33 Sämtliche Rechtsbehelfe des Datenschutzgesetzes setzen jedoch voraus, dass der Betroffene von der Datenbearbeitung Kenntnis hat, weshalb die Verletzung des Erkennbarkeitsgrundsatzes grundsätzlich als schwer zu betrachten ist.34 Zudem wird mittels Dashcams eine Vielzahl weiterer Personen (etwa Passanten) aufgezeichnet. Sodann besteht ein allgemeines gesellschaftliches Interesse an einem überwachungsfreien Zustand.35

Somit ist vorweg bei anlasslosen Dashcam-Aufzeichnungen davon auszugehen, dass kein überwiegendes Interesse an einer persönlichkeitsverletzenden Datenbearbeitung besteht. Erfolgt die Dashcam-Aufzeichnung zur eigenen Beweissicherung anlassbezogen und nur kurz, ist ein überwiegendes privates Interesse denkbar. 36

Vgl. Entscheid des Bezirksgerichts Schwyz SEO 201619 vom 20. Oktober 2016, E. 1.6.3.

33 Vorne Rz. 9.

34 Haag (Fn. 19), S. 179.

35 Vgl. Wermelinger, in: SHK-DSG (Fn. 16), Art. 13 Rz. 9; In Deutschland werden Bedenken geäussert, dass der Einsatz von Dashcams einen ständigen Überwachungsdruck verursacht und die Individuen deshalb ihr Verhalten ändern, weshalb in diesem Zusammenhang auch vom «Orwell'schen Überwachungsstaat» die Rede ist - in Anlehnung an den Roman «1984» von George Orwell, welcher einen totalitären Überwachungsstaat thematisiert (vgl. Niehaus Holger, Verwertbarkeit von Dashcam-Aufzeichnungen im Strafund Ordnungswidrigkeitenverfahren - zugleich Anmerkung zu Oberlandesgericht Stuttgart, Beschl. v. 04.05.2016 - 4 Ss 543/15, in: NZV 2016, S. 554 ff.; vgl. auch Cornelius Kai, Verwertung privat gefertigter Dashcam-Videos im VerkehrsBussgeldverfahren - zugleich Anmerkung zu Oberlandesgericht Stuttgart, Beschl. v. 04.05.2016 - 4 Ss 543/15, in NJW 2016, S. 2282); vgl. auch Haag (Fn. 19), S. 179 f.

36 So hat das Obergericht des Kantons Zug entschieden, dass im ihm vorliegenden Fall das Interesse des Dashcam-Verwenders an der

\section{Strafrechtliche Betrachtung}

20 Bei der Frage, ob eine DashcamAufzeichnung strafrechtswidrig erlangt wurde, dürfte regelmässig Art. 179quater StGB im Zentrum der Prüfung stehen. 37 In der überwiegenden Mehrzahl der Fälle ist dieser Straftatbestand durch das Filmen mittels Dashcams jedoch nicht erfüllt, da die Aufzeichnungen regelmässig öffentliche Strassen und nicht den Geheimbereich oder eine nicht ohne weiteres zugängliche Tatsache aus dem Privatbereich betreffen. 38

\section{Verwertbarkeit von Videoaufnahmen durch die Strafverfolgungsbehörden}

21 Wie noch zu zeigen sein wird, ist es für die Frage der Verwertbarkeit von privaten Dashcam-Aufzeichnungen zentral, ob

Beweissicherung höher zu gewichten sei als dasjenige des Gefilmten, sich unbeobachtet und unkontrolliert im Strassenverkehr zu bewegen. So habe die Aufnahme des Überfahrens einer doppelten Sicherheitslinie durch einen ersten deutlich gewichtigeren Verstoss (grobe Verletzung der Verkehrsregeln nach Art. 34 Abs. 4 SVG) einen Anlassbezug erhalten. In Bezug auf die anlasslosen Aufnahmen hielt das Obergericht fest, dass es zumindest problematisch erscheine, das Geschehen auf der Strasse ständig zu filmen. Es liess die Frage der Verwertbarkeit der anlasslosen Dashcam-Aufzeichnungen allerdings offen (Urteil Obergericht Zug S 201657 vom 11.05.2017, E. 4.5). Das Bundesgericht beanstandete dieses Urteil nicht, liess die Frage der Verwertbarkeit der Dashcam-Aufzeichnungen jedoch offen (Urteil des Bundesgerichts 6B_758/2017 vom 26. September 2017, insb. E. 1.4.3).

37 Gemäss Art. 179quater Abs. 1 StGB macht sich strafbar, «wer eine Tatsache aus dem Geheimbereich eines andern oder eine nicht jedermann ohne weiteres zugängliche Tatsache aus dem Privatbereich eines andern ohne dessen Einwilligung mit einem Aufnahmegerät beobachtet oder auf einen Bildträger aufnimmt».

38 Vgl. Entscheid des Bezirksgerichts Schwyz SEO 201619 vom 20. Oktober 2016, E. 1.5 sowie Entscheid des Kantonsgerichts Schwyz STK 20171 vom 20. Juni 2017, E. 3a. 
die Strafbehörden die umstrittenen Videoaufzeichnungen selbst rechtmässig hätten erlangen können. 39 Aus diesem Grund wird kurz erläutert, unter welchen Voraussetzungen die Strafbehörden dazu befugt sind, (insb. im Strassenverkehr) Videoaufnahmen zu erstellen.

Im Bereich der Videoüberwachung sind grundsätzlich die Kantone für die Regelung und Durchführung zuständig. Dient die Videoüberwachung allerdings nicht mehr (überwiegend) präventiven Zwecken, sondern wird an einem Tatverdacht angeknüpft, überwiegt der Zweck der Strafverfolgung und die Videoüberwachung wird abschliessend durch den Bund geregelt. 40

23 Gesetzliche Grundlage für Polizei und Staatsanwaltschaft für das Filmen im öffentlichen Raum (insb. auch im Strassenverkehr) bilden Art. 299 Abs. 1 und Abs. 2 lit. a StPO, sofern denn ein Anfangsverdacht besteht. ${ }^{41}$ Diese Bestimmungen statuieren, dass die Polizei und Staatsanwaltschaft im Vorverfahren ausgehend vom Verdacht, dass eine Straftat begangen worden sei - Beweise sammeln um festzustellen, ob gegen einen Beschuldigten ein Strafbefehl zu erlassen ist.

24 Gesetzliche Grundlage für sog. «Nachfahrkontrollen»42 bilden einerseits verschiedene Bestimmungen in der Strassenkontrollverordnung (SKV) sowie der Verordnung des ASTRA hierzu, ande-

39 Hinten Kapitel IV.3.a.

40 Zum Ganzen Müller (Fn. 10), S. 195 ff.

${ }^{41}$ Giger Hans, Analyse der Rechtsprobleme im Zusammenhang mit dem Beweisverwertungsverbot im Strassenverkehrsrecht, in: Strassenverkehr 1/2016, S. 7.

42 Vgl. Art. 6 lit. c Ziff. 2 VSKV-ASTRA. rerseits Normen der kantonalen Polizeigesetze. Die Kontrolle des Verkehrs auf öffentlichen Strassen obliegt gemäss Art. 3 Abs. 1 Satz 1 SKV der nach kantonalem Recht zuständigen Polizei, wobei gemäss Art. 9 Abs. 1 Satz 1 SKV nach Möglichkeit technische Hilfsmittel einzusetzen sind. 43

25 Fraglich - und soweit ersichtlich noch nicht bundesgerichtlich entschieden ist, ob bei der Nachfahrkontrolle oder bei einer stationären Geschwindigkeitskontrolle, bei welcher mittels Lasermesssystem und digitalem Videorecorder ununterbrochen Bilder erfasst werden, eine Anlasstat bzw. ein Anfangsverdacht vorausgesetzt wird, damit die Aufzeichnungen verwertet werden können. 44

26 Nach der hier vertretenen Auffassung ist ein Anfangsverdacht zu fordern. So dürfen präventiv-polizeiliche Kontrollmöglichkeiten (im Bereich der Verkehrssicherheit) nicht zur Umgehung strafprozessualer Schranken der Beweis-
$43 \overline{\text { Zum Ganzen vgl. Urteil des Bundesgerichts }}$ 6B_1025/2015 vom 4. November 2015, E. 2.3; sowie Entscheid des Kantonsgerichts St. Gallen ST.2015.34 vom 27. Oktober 2015, E. 2 b.

44 Aufgrund des Urteils des Bundesgerichts 6B_1004/2016 vom 14. März 2017 könnte man meinen, die Polizei benötige für eine Nachfahrkontrolle eine Anlasstat. Das Bundesgericht setzte sich allerdings nur praktisch mit der Videoaufzeichnung auseinander, ohne die Frage zu behandeln, ob es überhaupt eine Anlasstat braucht (vgl. E. 1.4.1); auch im Urteil des Bundesgerichts 6B_538/2015 vom 7. September 2015 setzte sich das Bundesgericht nur praktisch mit dem Anfangsverdacht auseinander und bejahte diesen, da die massive Geschwindigkeitsüberschreitung «auch ohne technische Hilfsmittel ohne Weiteres feststellbar [war]» (E. 1.2). 
sammlung missbraucht werden. 45 Ohne jeglichen Anfangsverdacht ergriffene strafprozessuale Massnahmen stellen regelmässig schwere Eingriffe in die dadurch betroffenen Grundrechte dar und sind infolgedessen dem Einzelnen grundsätzlich nicht zumutbar. ${ }^{46}$ Strafprozessual stellt eine anlasslose Beweiserhebung eine unzulässige Beweisausforschung («fishing expedition») dar. 47

Hinzuweisen ist an dieser Stelle auf den Umstand, dass das zufällige, auf einzelne Vorgänge beschränkte und kurzfristige Filmen eines Verkehrssünders durch die Polizei keine Observation im Sinne von Art. 282 f. StPO darstellt. Eine Observation gemäss der StPO liegt nur bei einem systematischen und dauerhaften Beobachten über einen längeren Zeitraum vor. 48

\section{Verwertbarkeit privat erhobener Dashcam-Aufzeichnungen}

\section{Beweisverwertungsverbote und privat beschaffte Beweise}

28 In der Strafprozessordnung nicht explizit geregelt ist, wieweit die Beweisverbote

45 Gless Sabine, in: Niggli/Heer/Wiprächtiger (Hrsg.), Basler Kommentar, Schweizerische Strafprozessordnung/Jugendstrafprozessordnung (Art. 1-195 StPO), 2. Aufl., Basel 2014 (nachfolgend zit.: Gless, in: BSK-StPO I), Art. 141 Rz. 38; Entscheid des Kantonsgerichts Schwyz STK 20171 vom 20. Juni 2017, E. 3b.aa.

46 Giger (Fn. 41), S. 7.

47 Entscheid des Kantonsgerichts Schwyz STK 2017 1 vom 20. Juni 2017, E. 3b.aa; zur «fishing expedition» vgl. BGE 137 I 218 E. 2.3.2 S. 222.

48 Vgl. Eugster Luzius/Katzenstein Annegret, in: Niggli/Heer/Wiprächtiger (Hrsg.), Basler Kommentar, Schweizerische Strafprozessordnung/Jugendstrafprozessordnung (Art. 196-457 StPO, Art. 1-54 JStPO), 2. Aufl., Basel 2014, Art. 282 Rz. 1.
Anwendung finden, wenn nicht staatliche Behörden, sondern Privatpersonen Beweismittel sammeln.49 In der Lehre ist die Frage strittig, ob bzw. wann Beweisverbote auch für beweiserhebende Private gelten sollen.50 Fraglich ist insbesondere, inwieweit die Beweisverwertungsverbote von Art. 141 StPO auch bezüglich der von Privaten gesammelten Beweise gelten. ${ }^{1}$

29 Nach herrschender Auffassung gilt kein prinzipielles Verwertungsverbot von durch Private rechtswidrig erlangten Beweisen. Von Privaten rechtswidrig beschaffte Beweismittel sind gemäss überwiegender Lehrmeinung dann verwertbar, wenn der Staat diese selbst auf rechtmässigem Wege hätte erlangen können und eine Interessenabwägung für ihre Verwertung spricht. ${ }^{2}$

49 Vgl. statt vieler Urteil des Bundesgerichts 6B_786/2015 vom 8. Februar 2016, E. 1.2; Art. 150 VE-StPO hielt noch fest, dass von Privaten in strafbarer Weise erlangte Beweise nur verwertet werden dürfen, «wenn das öffentliche oder private Interesse an der Wahrheitsfindung die durch die verletzten Strafbestimmungen geschützten Interessen überwiegt».

50 Gless, in: BSK-StPO I (Fn. 45), Art. 141 Rz. 40.

51 Schmid Niklaus/Jositsch Daniel, Handbuch des schweizerischen Strafprozessrechts, 3. Aufl., Zürich/St. Gallen 2017 (nachfolgend zit.: Schmid/Jositsch, Handbuch StPO), Rz. 802.

52 Gless, in: BSK-StPO I (Fn. 45), Art. 141 Rz. 40 ff.; Wohlers Wolfgang, in: Donatsch/Hansjakob/ Lieber (Hrsg.), Kommentar zur Schweizerischen Strafprozessordnung (StPO), 2. Aufl., Zürich/Basel/Genf 2014 (nachfolgend zit.: BearbeiterIn, in: StPO-Komm.), Art. 141 Rz. 8 f.; Häring Daniel, Verwertbarkeit rechtswidrig erlangter Beweise gemäss Schweizerischer Strafprozessordnung - alte Zöpfe oder substanzielle Neuerungen?, in: ZStrR 127/2009, S. 231 f.; Godenzi Gunhild, Private Beweisbeschaffung im Strafprozess: Eine Studie zu strafprozessualen Beweisverboten im schweizerischen und deutschen Recht, Diss. Univ. Zürich, Zürich/Basel/Genf 2008 (nachfolgend zit.: Godenzi, Diss.), S. 264 ff. und 341 f.; dies., Strafbare Beweisverwertung? Auswirkungen der Art. $179^{\text {bis }}$ ff. StGB auf die Beweisverwertung im Strafverfahren, in: AJP 2012 
30 Die hypothetische Voraussetzung, wonach die Strafverfolgungsbehörden das Beweismittel auf rechtmässigem Weg hätten erlangen können müssen, wird in der Lehre allerdings auch kritisiert. So bleibe eine entsprechende Überprüfung einerseits nur hypothetisch, andererseits schaffe sie mit Bezug auf das staatliche Strafmonopol (Art. 2 Abs. 1 StPO) auch falsche Anreize zur detektivischen Eigeninitiative.53 Zudem seien Beweisergebnisse zwar oft auch auf rechtmässigem Weg beschaffbar, jedoch wäre ohne das Delikt des Privaten der Fall den Strafverfolgungsbehörden gar nicht bekannt geworden. 54

31 Andere Kommentatoren fordern, dass eine Verwertung von durch Private strafrechtswidrig erhobenen Beweisen nur zugelassen werden sollte, wenn dies - wie von Art. 141 Abs. 2 StPO für den Staat vorgesehen - zur Aufklärung schwerer Straftaten unerlässlich ist. 55

(nachfolgend zit.: Godenzi, AJP 2012), S. 1253 f.; Schmid Niklaus/Jositsch Daniel, Praxiskommentar zur Schweizerischen Strafprozessordnung, 3. Aufl., Zürich/St. Gallen 2018 (nachfolgend zit.: Schmid/Jositsch, PraxKomm-StPO), Art. 141 Rz. 3; dies., Handbuch StPO (Fn. 51), Rz. 802; Bénédict Jérôme/Treccani Jean, in: Kuhn/ Jeanneret (Hrsg.), Commentaire Romand, Code de procédure pénale suisse, Basel 2011 (nachfolgend zit.: Bénédict/Treccani, in: CR-CPP), Intro Art. 139-141 Rz. 12; Wohlers Wolfgang/Bläsi Linda, Dogmatik und praktische Relevanz der Beweisverwertungsverbote im Strafprozessrecht der Schweiz, in: recht 2015, S. 162.

53 Riedo Christof/Fiolka Gerhard/Niggli Marcel Alexander, Strafprozessrecht sowie Rechtshilfe in Strafsachen, Basel 2011, § 30 Rz. 1080.

54 Riklin Franz, Orell Füssli Kommentar, Schweizerische Strafprozessordnung mit JStPO, StBOG und weiteren Erlassen, 2. Aufl., Zürich 2014, Art. 141 Rz. 4.

55 Jositsch Daniel, Grundriss des schweizerischen Strafprozessrechts, 3. Aufl., Zürich/St. Gallen 2017, § 58 Rz. 285; Müller (Fn. 10), S. 356.
32 Die gefestigte Rechtsprechung des Bundesgerichts lehnt sich an die überwiegende Lehrmeinung an. Demnach sind «von Privaten rechtswidrig erlangte Beweismittel nur verwertbar, wenn sie von den Strafverfolgungsbehörden rechtmässig hätten erlangt werden können und kumulativ dazu eine Interessenabwägung für deren Verwertung spricht».56

\section{Zivil- und datenschutzrechtswidrig erhobene Dashcam-Aufzeichnungen}

33 Von zentraler Bedeutung für die Verwertbarkeit privater Dashcam-Aufzeichnungen ist die Frage, wie es sich verhält, wenn Private bei der Beweisbeschaffung nicht gegen eine strafrechtliche Bestimmung, sondern «nur» gegen eine ziviloder datenschutzrechtliche Norm verstossen. 57

34 Ein Grossteil der Lehre geht im Zusammenhang mit der Verwertbarkeit privater Beweise überhaupt nicht bzw. nicht explizit auf «schlicht rechtswidrige» Beweismittel ein.

35 Folgt man dem Rechtswidrigkeitsbegriff von Fornito, kann Rechtswidrigkeit nur bestehen oder fehlen.58 Konsequenterweise wären nach dieser Definition der Rechtswidrigkeit damit «schlicht rechtswidrige» Beweise ebenso erfasst wie

56 Vgl. statt vieler Urteil des Bundesgerichts 6B_1241/2016 vom 17. Juli 2017, E. 1.2.2; oder Urteil des Bundesgerichts 1B_76/2016 vom 30. März 2016, E. 2.2; auf diese beiden Voraussetzungen wird in Kapitel IV.3 im Detail eingegangen.

57 Wie gesehen dürfte der Einsatz von Dashcams häufig keinen Straftatbestand erfüllen (Kapitel II.3), hingegen regelmässig eine Verletzung des Persönlichkeits- sowie des Datenschutzes darstellen (Kapitel II.1 und II.2).

58 Fornito Roberto, Beweisverbote im schweizerischen Strafprozess, Diss. Univ. St. Gallen, St. Gallen 2000, S. 288. 
strafrechtswidrige Beweismittel. Gless differenziert bei der Frage der Verwertbarkeit privater Beweismittel zwar zwischen rechtmässigen, rechtswidrigen und strafbaren Privatermittlungen, erachtet mit Blick auf die neuere Rechtsprechung die Voraussetzungen für eine Verwertbarkeit in den beiden letztgenannten Fällen aber als identisch.59

36 Gegen ein strafprozessuales Abwägungsverfahren bei «schlicht (zivil-) rechtswidrigen» Rechtsverstössen durch Private spricht sich Godenzi aus. ${ }^{60}$ Sie führt aus, dass je weiter der Kreis der Rechtsverstösse gezogen wird, welche ins strafprozessuale Beweisrecht durchschlagen sollen, desto eher der Staat mit einem Verwertungsverbot dafür einstehen muss, dass ihm private Ermittler bei der Aufklärung einer Straftat mit rechtswidrigen Machenschaften «reinpfuschen».61 Es sei nicht Aufgabe des Staates, in einem Strafverfahren mithilfe von strafprozessualen Verwertungsverboten von Amtes wegen die Gutheissung oder Ablehnung eines Zivilrechtsverstosses auszusprechen. Die Verantwortung für die Bewältigung schlicht zivilrechtswidriger Vorgänge obliege den Zivilparteien.62 Auch Riedo/Fiolka/Niggli sind der Auffassung, dass Widerhandlungen gegen rein zivilrechtliche Regelungen im Zusammenhang mit der Verwertbarkeit privat

59 Gless, in: BSK-StPO I (Fn. 45), Art. 141 Rz. 4Oc.

60 Godenzi Gunhild, Korruptionsaufklärung durch Unternehmen - ein Fluchtweg ins Dunkelfeld, in: Ackermann/Wohlers (Hrsg.), Korruption in Staat und Wirtschaft, 4. Zürcher Tagung zum Wirtschaftsrecht, Zürich 2010 (nachfolgend zit.: Godenzi, Korruptionsaufklärung), S. 183; dies., Diss. (Fn. 52), S. 170 f. und 195 f.

61 Godenzi, Korruptionsaufklärung (Fn. 60), S. $183 \mathrm{f}$.

62 Godenzi, Diss. (Fn. 52), S. 170. beschaffter Beweise ohne Belang bleiben müssen. 63

37 Für eine strafprozessuale Relevanz datenschutzrechtlicher Aspekte sprechen sich hingegen Jöhri/Studer aus. Sie fordern, dass der durch das Zivil- und Verwaltungsrecht gewährleistete Persönlichkeitsschutz auch im Rahmen von Strafverfahren berücksichtigt werden müsse. ${ }^{64}$ Ebenfalls spricht sich Müller im Zusammenhang mit der Verwertung von zivilrechts- bzw. datenschutzrechtswidrig erhobenen Beweisen aus einer Videoüberwachung für eine Abwägung zwischen dem Interesse an der Wahrheitsfindung und der Strafverfolgung gegenüber dem Interesse des Betroffenen, dass seine Daten nicht in persönlichkeitsverletzender Weise bearbeitet werden, aus. ${ }^{65}$ Dieser Meinung sind wohl auch Hansjakob und Häring. 66

38 Habscheid - noch zum alten Recht wendet sich gegen die Meinung, wonach die Zulässigkeit von rechtswidrig, insbesondere unter Verletzung des Persönlich-

$63 \overline{\text { Solange sich der Private nicht strafbar mache, }}$ tangiere etwa eine Persönlichkeitsverletzung (Art. 28 ZGB) die Beweisverwertbarkeit nicht (Riedo/Fiolka/Niggli [Fn. 53], § 30 Rz. 1078).

64 Jöhri Yvonne/Studer Marcel, Entscheidanmerkung zum Urteil des Bundesgerichts 6B_536/2009 vom 12. November 2009, in: forumpoenale 3/2010, S. $160 \mathrm{f}$.

65 Müller (Fn. 10), S. 357.

66 Ersterer hält fest, dass die Frage der Verwertbarkeit von Ergebnissen aus unzulässigen privaten Überwachungen (etwa Kameras in Warenhäusern) anhand einer Interessenabwägung zu entscheiden sei (Hansjakob, in: StPO-Komm. [Fn. 52], Art. 280 Rz. 6). Letzterer spricht sich für eine Interessenabwägung aus, nach welcher durch Private rechtswidrig erlangte Beweise grundsätzlich nur dann verwertbar seien, wenn bei ihrer Beschaffung kein Rechtsgut verletzt wurde, welches im konkreten Fall Vorrang vor dem Interesse an der Wahrheitsfindung sowie an der Durchsetzung des Strafrechts hat (Häring [Fn. 52], S. 232). 
keitsrechts erlangten Beweismitteln von Fall zu Fall zu entscheiden sei. Gemäss seiner Auffassung darf im Prozess nicht als legitimer Beweis zugelassen werden, was strafrechtlich oder zivilrechtlich Unrecht ist. ${ }^{67} \mathrm{Er}$ betont, dass es im Strafprozess nicht um die Durchsetzung und Ermittlung der Wahrheit um jeden Preis gehe, sondern um die Wahrheitsfindung in einem rechtsstaatlich geordneten Verfahren. Ansonsten wäre die stärkere und rücksichtslosere Partei im Vorteil. ${ }^{68}$

39 Der in der Lehre geäusserten Auffassung, wonach rein zivilrechtliche Normverstösse bei der Verwertbarkeit privat beschaffter Beweise unberücksichtigt bleiben müssen, kann nicht gefolgt werden. Entscheidend ist nicht, ob es sich bei der verletzten Norm um eine Bestimmung des Strafrechts oder etwa des Datenschutzrechts handelt, sondern vielmehr, dass sie dem Persönlichkeitsschutz des Betroffenen dient. ${ }^{69}$ Auch ist nicht ersichtlich, weshalb das zwingende Zivilrecht immer nur zwischen den Parteien selbst wirken soll.70

67 Habscheid Walther J., Beweisverbot bei illegal, insbesondere unter Verletzung des Persönlichkeitsrechts, beschafften Beweismitteln, in: SJZ 89/1993, S. 185 f.

68 Habscheid (Fn. 67), S. 189.

69 So in der deutschen Literatur Niehaus (Fn. 35), S. $552 \mathrm{f}$.

70 So sorgen die Behörden etwa dafür, dass die Grundrechte - etwa das in Art. 13 Abs. 2 BV verankerte Recht auf informationelle Selbstbestimmung - mittels indirekter Drittwirkung auch unter Privaten wirksam werden, was etwa im Rahmen einer Interessenabwägung geschieht (vgl. Gächter Thomas, in: Biaggini/Gächter/Kiener [Hrsg.], Staatsrecht, 2. Aufl., Zürich/ St. Gallen 2015, § 30 Rz. 59 ff.; Müller [Fn. 10], S. 308 ff.). Zudem sind bei einer Persönlichkeitsverletzung unter Privaten zugleich häufig auch öffentliche Interessen tangiert. Wird etwa jemand im öffentlichen Verkehr mittels einer Dashcam gefilmt, stellt dies einerseits regelmässig eine Verletzung des Rechts am eigenen Bild
40 Ebenfalls ist zwar denkbar, dass Private die Behörden mit eigenen rechtswidrigen Beweisermittlungen behindern. Der umgekehrte Fall, wonach sich die Behörden eines privaten Beweises bedienen, um dadurch erst ein Strafverfahren einleiten zu können, dürfte jedoch deutlich häufiger vorkommen. Damit besteht kein wesentlicher Unterschied mehr, ob die Beweiserhebung durch Private oder durch die Behörden erfolgt, weshalb auch zivil- und datenschutzrechtliche Regelungen berücksichtigt werden müssen.

41 Nicht gefolgt werden kann schliesslich der Meinung von Habscheid. ${ }^{71}$ Einerseits stellt ein generelles Verwertungsverbot solcher Beweise eine Überspitzung der Formvorschriften zulasten der Verbrechensaufklärung dar, 72 anderseits kann mittels Interessenabwägung ein Ausgleich geschaffen und das Verhalten der rücksichtslosen Partei entsprechend berücksichtigt werden.

42 Das Bundesgericht musste sich mit Urteil 6B_1310/2015 vom 17. Januar 2017 - soweit ersichtlich - erstmals mit der Verwertung eines Beweismittels auseinandersetzen, das ein Privater zwar allgemein rechtswidrig (Persönlichkeitsverletzung nach Art. $28 \mathrm{ZGB}$ ), nicht aber deliktisch erlangt hatte. Es schützte die vorinstanzliche Anwendung von Art. 141 Abs. 2 StPO sowie des bundesgerichtlichen Schemas zu von Privaten rechtswidrig erlangten Beweismitteln auch bei einem allgemein widerrechtlichen Be-

der gefilmten Person dar, andererseits wird dadurch auch das öffentliche Interesse verletzt, in öffentlich zugänglichen Räumen nicht ständig beobachtet bzw. überwacht zu werden.

${ }_{71}$ Vorne Rz. 38.

72 Häring (Fn. 52), S. 231. 
weismittel.73 Das Bundesgericht stellte damit «schlicht rechtswidrige» Beweise den strafrechtswidrigen Beweismitteln im Hinblick auf sein Prüfschema gleich.

Diese Vorgehensweise wurde bereits vor dem genannten Bundesgerichtsentscheid etwa durch das Obergericht des Kantons Bern praktiziert.74

43 Im Ergebnis ist damit bei «schlicht rechtswidrigen» Dashcam-Aufzeichnungen das gleiche Prüfschema anzuwenden wie bei strafrechtswidrigen Dashcam-Aufzeichnungen.

\section{3. (Straf-)rechtswidrig erlangte Dashcam-Aufzeichnungen}

44 Damit ist allerdings noch nichts darüber gesagt, was unter den Kriterien zu verstehen ist, welche das Bundesgericht in seinem Prüfschema zu rechtswidrigen privaten Beweisen anwendet. Diese werden im Folgenden eingehend behandelt.

\section{a) Hypothetisch rechtmässige Beweiserlangung durch die Strafbehörden}

45 Wie gesehen verlangt das bundesgerichtliche Prüfschema zunächst, dass die Strafverfolgungsbehörden das umstrittene Beweismittel selbst rechtmässig hätten erlangen können. 75

46 Entscheidend für die hypothetisch rechtmässige Beweiserlangung ist gemäss Bundesgericht, ob die Strafbehör-

73 Urteil des Bundesgerichts 6B_1310/2015 vom 17. Januar 2017, E. 7.

74 Entscheid des Obergerichts des Kantons Bern SK 2013275 vom 1. Mai 2014 im Zusammenhang mit einer «schlicht rechtswidrigen" Videoüberwachung in einem Erlebnisbad.

75 Vorne Kapitel IV.1; zu der für die Verwertbarkeit zusätzlich erforderlichen Interessenabwägung vgl. hinten Kapitel IV.3.b. den das umstrittene Beweismittel rechtmässig hätten beschaffen können, «wenn ihnen der Tatverdacht bekannt gewesen wäre». 76

47 Es führt aus, dass betreffend die Frage, ob die Strafverfolgungsbehörden das fragliche Beweismittel rechtmässig hätten erlangen können, «nur solche gesetzlichen Erfordernisse einzubeziehen [sind], die sich abstrakt anwenden lassen und keine Würdigung konkreter Umstände der jeweiligen Beweiserlangung erfordern». 77

48 Es scheint so, als ob das Bundesgericht bei der Frage, ob die Strafbehörden den umstrittenen Beweis hätten rechtmässig beschaffen können, den Tatverdacht der definitionsgemäss von konkreten Umständen abhängt -78 nicht nachprüft. In der Vergangenheit prüfte es im $\mathrm{Zu}-$ sammenhang mit der hypothetischen Erlangung privater Ton- und Videoaufzeichnungen wiederholt nur, ob eine Katalogtat nach Art. 269 Abs. 2 lit. a StPO vorlag und die Behörden damit zu den betreffenden Beweiserhebungen (Überwachungsmassnahmen nach Art. 269 und 280 StPO) befugt gewesen wären.

$76 \overline{\text { Urteil des Bundesgerichts 6B_1241/2016 vom }}$ 17. Juli 2017, E. 1.2.2; Urteil des Bundesgerichts 6B_786/2015 vom 8. Februar 2016, E. 1.3.1; Urteil des Bundesgerichts 6B_983/2013 vom 24. Februar 2014, E. 3.3.1.

77 Urteil des Bundesgerichts 6B_786/2015 vom 8. Februar 2016, E. 1.3.1 mit Hinweis auf Godenzi, Diss. (Fn. 52), S. $315 \mathrm{ff}$.

78 Als Tatverdacht gilt gemäss bundesgerichtlicher Rechtsprechung «der Nachweis konkreter Verdachtsmomente, wonach das inkriminierte Verhalten den fraglichen Tatbestand erfüllen könnte». Dieser muss sich dabei aus konkreten Tatsachen ergeben, die eine vorläufige Subsumtion unter einen bestimmten Tatbestand erlauben (Urteil des Bundesgerichts 6B_694/2011 vom 23. Januar 2012, E. 3.3; Urteil des Bundesgerichts 1B_516/2011 vom 17. November 2011, E. 2.1 m.w.H.). 
Den Tatverdacht (sowie den Subsidiaritätsgrundsatz) überprüfte es hingegen wiederholt nicht ausdrücklich.79

Zwar hielt das Bundesgericht im Urteil 1B_22/2012 vom 11. Mai 2012 fest, dass die Vorinstanz ohne Bundesrechtsverletzung annehmen durfte, die Strafverfolgungsbehörden hätten die umstrittene Videoaufzeichnung nicht selbst erlangen können, da zum Zeitpunkt ihrer Erstellung kein dringender Tatverdacht bestand. 80 Es präzisierte im Urteil 6B_983/2013 vom 24. Februar 2014 den Entscheid allerdings dahingehend, dass es im Zeitpunkt der fraglichen Beweisbeschaffung an einer Straftat überhaupt fehlte, und nicht nur an der strafbehördlichen Kenntnis des entsprechenden Tatverdachts. ${ }^{81}$

50 Das Bezirksgericht Schwyz bejahte im Zusammenhang mit der Verwertung einer Dashcam-Aufzeichnung die hypothetisch rechtmässige Erlangung des Beweismittels durch die Strafverfolgungsbehörden, da die Polizei aufgrund der in der Strassenkontrollverordnung enthaltenen verkehrspolizeilichen bzw. präventiv-polizeilichen Aufgaben berechtigt sei, das Verhalten der Strassenverkehrsteilnehmer mit technischen Hilfsmitteln etwa einer Kamera - aufzuzeichnen, ohne dass sie einen konkreten Tatverdacht

$79 \overline{\text { Vgl. Urteil des Bundesgerichts 6B_786/2015 vom }}$ 8. Februar 2016, E. 1.3.1 sowie Urteil des Bundesgerichts 6B_983/2013 vom 24. Februar 2014, E. 3.3.1.

80 Urteil des Bundesgerichts 1B_22/2012 vom 11. Mai 2012, E. 2.4.4.

81 Urteil des Bundesgerichts 6B_983/2013 vom 24. Februar 2014, E. 3.3.1 mit Hinweis auf Urteil des Bundesgerichts 1B_22/2012 vom 11. Mai 2012; Dabei ging es um Videoaufzeichnungen, welche ein Privater nicht nur vor einer Strafanzeige gemacht hatte, sondern bevor die zu beweisende Straftat überhaupt begangen wurde. benötige. Selbst wenn man davon ausginge, dass die Polizei im Sinne von Art. 197 Abs. 1 lit. b StPO nur aufgrund eines hinreichenden Tatverdachts Aufnahmen hätte machen dürfen, hätten die Strafverfolgungsbehörden die Aufnahmen erlangen können, da sie bereits aufgrund der Geschwindigkeit des Personenwagens konkrete Verdachtsmomente für strafbares Verhalten gehabt hätten und die Aufzeichnung nach Kenntnisnahme dieser Verdachtsmomente hätten starten können.82

51 Das Kantonsgericht Schwyz als Berufungsgericht hielt fest, dass nicht davon ausgegangen werden könne, dass die Beweismittel durch die zuständigen Strafverfolgungsbehörden hätten erlangt werden dürfen. Als der Fahrlehrer seine Dashcam einschaltete, fehlte es an einer Straftat und es bestand kein Anlass zu einer Kontrolle, in deren Rahmen die Polizei hätte Verdacht schöpfen, einem allfälligen Verdächtigen mit eingeschaltetem Videogerät nachfahren und diesen eruieren können. Sollten Polizeipatrouillen unterwegs verdeckt ohne konkreten Verdacht flächendeckend und anlasslos ständig filmen, stelle dies eine unzulässige «fishing expedition» dar. ${ }^{83}$

52 Ein Grossteil der Lehre befürwortet zwar, dass von Privaten (straf-)rechtswidrig erlangte Beweise im Strafverfahren zunächst nur zuzulassen sind, wenn diese von den Strafbehörden rechtmässig hätten erlangt werden können, jedoch thematisieren die wenigsten Kommenta-

$82 \overline{\text { Entscheid des Bezirksgerichts Schwyz SEO } 2016}$ 19 vom 20. Oktober 2016, E.1.6.

83 Entscheid des Kantonsgerichts Schwyz STK 2017 1 vom 20. Juni 2017, E. 3.b.aa; zur «fishing expedition» vgl. vorne Rz. 26. 
toren die Voraussetzungen für dieses Kriterium tiefergehend.

Godenzi hält fest, dass die Zulässigkeit des gedachten hypothetischen Eingriffs nach illegalen privaten Tonbandaufnahmen ausschliesslich vom Vorliegen einer Katalogtat und einer abstrakten Verhältnismässigkeit abhängt, wohingegen der Tatverdacht sowie der Subsidiaritätsgrundsatz im Rahmen der massgeblichen abstrakten Hypothese unberücksichtigt bleiben.84 Dies begründet sie damit, dass der Subsidiaritätsgrundsatz und der vorbestehende Tatverdacht untrennbar mit den konkreten Umständen des Einzelfalls verbunden seien. Konkrete Umstände des Einzelfalls seien jedoch nur bezogen auf die tatsächlich erfolgte private Ermittlungsmassnahme existent, nicht aber bei einer nachträglichen, nur gedachten staatlichen Überwachungsmassnahme. 85

54 Auch Schmid hält - mit Blick auf die Erwägungen des Bundesgerichts - fest, dass für die Beurteilung, ob die Behörden das gleiche Beweismittel rechtmässig hätten erlangen können, davon auszugehen sei, dass im Zeitpunkt der privaten Beweisbeschaffung bereits ein dringender Tatverdacht bestanden habe. Anhand dieser Hypothese sei zu prüfen, ob die Strafbehörden das gleiche Beweismittel hätten beschaffen dürfen. Folge man der bundesgerichtlichen Rechtsprechung, sei bei privaten Beweiserhebungen nicht von Belang, ob der Verdacht ein begründeter sei bzw. ob überhaupt ein Verdacht vor-

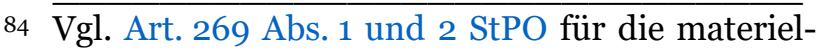
len Voraussetzungen, gemäss welchen die Staatsanwaltschaft den Post- und Fernmeldeverkehr überwachen lassen kann.

85 Godenzi, Diss. (Fn. 52), S. 311 ff. und insb. S. $315 \mathrm{f}$. liege. Die Hürden für die Annahme einer rechtmässigen Beweismittelerlangung seien bei Privaten um einiges tiefer angelegt als beim Staat. ${ }^{86}$

55 Rusch hält fest, dass das Kriterium der hypothetischen Beweiserlangung im Strassenverkehr nur funktioniere, wenn man das Wissen der Behörden um den Tatverdacht der sich innert Sekunden ereignenden Tat fingiere, was das Bundesgericht tatsächlich so $\mathrm{zu}$ verstehen scheint. 87

56 Wie die Entscheide des Bezirksgerichts und des Kantonsgerichts Schwyz zeigen, ist für die Frage, ob die Strafbehörden die umstrittenen Videoaufzeichnungen rechtmässig hätten erlangen können, namentlich der Tatverdacht von zentraler Bedeutung.

57 Zwar scheint es, als beurteile das Bundesgericht den Tatverdacht im Rahmen der abstrakten Hypothesenprüfung nicht. Nichtsdestotrotz blendet es den Tatverdacht nicht gänzlich aus. Wie gesehen verneinte das Bundesgericht die Verwertbarkeit eines privaten Beweismittels (infolge fehlenden Tatverdachts) in einem Fall, wo es im Zeitpunkt der privaten Beweisbeschaffung an einer Straftat überhaupt fehlte. Der Private konnte somit also überhaupt keinen Tatverdacht haben.

86 Schmid Linda, Entscheidanmerkung zum Urteil des Bundesgerichts 6B_786/2015 vom 8. Februar 2016, in: forumpoenale 1/2017, S. $4 \mathrm{ff}$.

87 Rusch Arnold F., Little Red Corvette, Big Black Box, in: AJP 2016, S. 404 mit Hinweis auf Urteil des Bundesgerichts 6B_786/2015 vom 8. Februar 2016, E. 1.3.1; sowie Urteil des Bundesgerichts 6B_983/2013 vom 24. Februar 2014, E. 3.3.1. 
58 Für die Frage der hypothetisch rechtmässigen Erreichbarkeit privater Dashcam-Aufzeichnungen drängt sich deshalb eine Unterscheidung zwischen anlassbezogenen und anlasslosen privaten Dashcam-Aufzeichnungen auf.

Anlassbezogene private Dashcam-Aufzeichnungen - also Videoaufzeichnungen, welche ein Privater aufgrund konkreter Verdachtsmomente hinsichtlich einer Straftat erstellt - sind in jedem Falle auch hypothetisch durch die Strafbehörden rechtmässig zu erlangen.

60 Bei anlasslosen Dashcam-Aufzeichnungen - sprich bei Videoaufnahmen von permanent aufzeichnenden Dashcams - ist hingegen zu differenzieren.

61 Wo sich das gefilmte Verhalten auf einen Moment konzentriert, welcher nur mit Daueraufnahme hat erfasst werden können, müsste auch eine abstrakte Beurteilung des Tatverdachts zu einer Verneinung der Erreichbarkeit führen. Wie gesehen stellen Beweisaufnahmen durch die Strafbehörden ohne (genügenden) Tatverdacht eine unzulässige Beweisausforschung (fishing expedition) dar. ${ }^{88}$ Demnach scheitert in solchen Konstellationen die Verwertbarkeit der privaten Dashcam-Aufzeichnung daran, dass die Strafbehörden diese nicht selbst hätten rechtmässig erlangen können. ${ }^{89}$

88 Vorne Rz. 26.

89 Die gescheiterte Beweishypothese fungiert hierbei bei Privaten also als absolute Schranke der Wahrheitsfindung, welche sich auch durch eine Interessenabwägung nicht relativieren lässt (vgl. Godenzi, AJP 2012 [Fn. 52], S. 1246); demgegenüber hat das Bundesgericht die Unzulässigkeit einer (behördlichen) «fishing expedition» zwar bejaht, es liess die Frage, ob die Ergebnisse einer solchen unzulässigen Beweisausforschung
62 Hingegen muss die rechtmässige Erlangung anlassloser Videoaufzeichnungen in Fällen bejaht werden, wo die Polizei wäre sie anstelle des privaten DashcamVerwenders vor Ort gewesen - nach ersten Verdachtsmomenten noch eine Kamera hätte einschalten können. ${ }^{90}$

\section{b) Interessenabwägung}

63 Führt die Hypothesenprüfung zum Ergebnis, dass eine privat beschaffte Dashcam-Aufzeichnung durch die Strafbehörden rechtmässig hätte beschafft werden können, muss eine Interessenabwägung vorgenommen werden.91 Solche Interessenabwägungen sind bekanntermassen immer mit Rechtsunsicherheiten verbunden. ${ }^{2}$

64 Die Interessen des Staates an der Bestätigung oder Widerlegung des konkreten Verdachts und die Interessen des Betroffenen an der Wahrung seiner Persönlichkeitsrechte sind gegeneinander

absolut oder nur relativ unverwertbar sind, allerdings offen (vgl. BGE 137 I 218 E. 2.3.2 S. 222 f.).

90 Mäder (Fn. 30) gelangt zum Schluss, dass die hypothetisch rechtmässige Erreichbarkeit einer Dashcam-Aufnahme zu verneinen sei, wenn es nur um ein einzelnes Delikt ohne vorangehendes, den hypothetischen Verdacht begründendes Verhalten geht. Im Strassenverkehr heisse dies konkret, dass erst nach der ersten beobachteten Verkehrsregelverletzung die Strafverfolgungsbehörden rechtmässig hätten aufzeichnen dürfen (S. 166). Dem ist grundsätzlich beizupflichten. Allerdings sind auch Konstellationen denkbar, bei welchen die Strafbehörden - wären sie anstelle des Privaten vor Ort gewesen - bereits während der ersten Verkehrsregelverletzung aufgrund der Dauer des Delikts (man denke etwa an eine mehrminütige Raserfahrt) konkrete Verdachtsmomente gehabt hätten und damit rechtmässig hätten aufzeichnen dürfen.

91 Eine "carte blanche» liegt in der abstrakten Hypothesenprüfung damit nicht vor (Schmid [Fn. 86], S. 5; vgl. auch Urteil des Bundesgerichts 6B_983/2013 vom 24. Februar 2014, E. 3.3.1).

92 Vgl. etwa Fornito (Fn. 58), S. 249 f., 252 f. 
abzuwägen, wozu alle erheblichen Umstände in Betracht zu ziehen sind.93

Die Art und Durchführung der durch das Bundesgericht vorgenommenen Interessenabwägung im Zusammenhang mit Beweisverwertungsverboten wurde in der Lehre wiederholt kritisiert.94 So wird angeführt, dass die Interessenabwägung unvollständig vorgenommen 95 oder lediglich formelhaft erwähnt wird. ${ }^{96}$ Ferner weist jede Interessenabwägung ein gewisses willkürliches Element auf,97 sodass jedes beliebige Resultat letztlich formaljuristisch korrekt «herbeigezaubert» werden kann. ${ }^{8}$ Es wird deshalb gefordert, dass die Interessenabwägung, der Methodenehrlichkeit folgend, transparent vorgenommen, die verschiedenen sich gegenüberstehenden Interessen vollständig erfasst, offengelegt und im Detail gegeneinander abgewogen werden. 99

Bei der Interessenabwägung zunächst zu berücksichtigen ist die Schwere des Tatvorwurfs. ${ }^{100}$

93 BGE 109 Ia 244 E. 2b S. 246 = Pra 72 (1983) Nr. 275.

94 Zwar betrifft diese Kritik nicht im Speziellen die Interessenabwägung, welche im Zusammenhang mit von Privaten rechtswidrig beschafften Beweisen durchgeführt wird, sie lässt sich aber grundsätzlich auf diese übertragen, da diesbezüglich die gleichen Unzulänglichkeiten feststellbar sind.

95 Etwa indem dem staatlichen Verfolgungsinteresse lediglich allfällige Individualinteressen (i.d.R. des Beschuldigten) gegenübergestellt werden.

96 Vest Hans/Höhener Andrea, Beweisverwertungsverbote - quo vadis Bundesgericht?, in: ZStrR 127/2009, S. 102 f.; vgl. auch Vest Hans/Eicker Andreas, Entscheidanmerkung, Bundesgericht, I. Öffentlichrechtliche Abteilung, 18.5.2004, Y. c. X. und Staatsanwaltschaft sowie Obergericht des Kantons Aargau, staatsrechtliche Beschwerde (BGE 130 I 126), S. 890.

97 Häring (Fn. 52), S. 245.

98 Fornito (Fn. 58), S. 252.

99 Vest/Höhener (Fn. 96), S. 102 f.

100 So etwa Vest/Höhener (Fn. 96), S. 99; Dabei gilt gemäss Bundesgericht: Je schwerer die zu beur-
67 Als «schwere Straftaten» kommen vorab Verbrechen (i.S.v. Art. 10 Abs. 2 StGB), Delikte der Schwerkriminalität (bei denen als Strafe ausschliesslich eine Freiheitsstrafe angedroht ist) sowie Delikte gemäss den Deliktskatalogen (etwa Art. 269 Abs. 2 StPO) in Frage. ${ }^{101}$ Demnach stellen insbesondere grobe Verkehrsregelverletzungen (Art. 90 Abs. 2 SVG) als Vergehen zwar relativ schwerwiegende nicht aber schwere Straftaten im genannten Sinne dar. ${ }^{102}$

68 Ein Teil der Lehre fordert zudem, dass nicht nur auf die abstrakte Strafandrohung, sondern auch auf die konkrete Schwere der Tat ${ }^{103}$ mit ihren konkreten Folgen ${ }^{104}$ abzustellen ist. ${ }^{105}$

69 Ebenfalls wird gefordert, dass - analog zur Rechtsprechung zu rechtswidrig erlangten Beweisen durch die Strafverfolgungsbehörden - Gewicht und Ausmass der Rechtsgüterverletzung bei der

teilende Straftat ist, desto eher überwiegt das öffentliche Interesse an der Wahrheitsfindung das private Interesse des Beschuldigten daran, dass der fragliche Beweis unverwertet bleibt (Urteil des Bundesgerichts 6B_323/2013 vom 3. Juni 2013, E. 3.5). In der Lehre wird allerdings häufig die gegenteilige Auffassung vertreten: Je schwerer das vorgeworfene Delikt, desto genauer müssen die rechtsstaatlichen Beweiserhebungsregeln beachtet werden (statt vieler Rusch [Fn. 87], S. 404).

101 Zum Ganzen Wohlers, in: StPO-Komm. (Fn. 52), Art. 141 Rz. 21a m.w.H.

102 Vgl. BGE 137 I 218 E. 2.3.5.2 S. 224; im Ergebnis auch Entscheid des Kantonsgerichts Schwyz STK 20171 vom 20. Juni 2017, E. 4b; ebenso Uttinger Ursula, Nutzung von Dashcam als Beweismittel, in: Jusletter vom 12. Februar 2018, Rz. 13.

103 Mäder (Fn. 30), S. 161 (insb. Fn. 61) und 166; Schmid (Fn. 86), S. 5.

104 So in der deutschen Literatur Niehaus (Fn. 35), S. 554.

105 Dem ist beizupflichten. So beträgt etwa der Strafrahmen, den Art. 139 Ziff. 1 StGB auch für den Diebstahl eines verschlossenen Fahrrades vorsieht, bis zu 5 Jahre. 
Beweisbeschaffung durch Private ebenso gewürdigt werden, da ansonsten der privaten Beweisbeschaffung Tür und Tor geöffnet würde und etwa auch durch ein Verbrechen erlangte private Beweismittel verwertet werden dürften. ${ }^{106}$ Dies geht einher mit dem ebenfalls miteinzubeziehenden Interesse des Beschuldigten an der Wahrung seiner Persönlichkeitsrechte. ${ }^{107}$ Hierbei zu berücksichtigen ist, dass durch Dashcams regelmässig das Recht auf informationelle Selbstbestimmung, das Recht am eigenen Bild sowie grundlegende Prinzipien des Datenschutzes verletzt werden.108 Demnach sind auch Gewicht und Ausmass der Persönlichkeitsverletzung durch den Dashcam-Verwender in die Interessenabwägung einzubeziehen. ${ }^{109}$

In der Interessenabwägung ist sodann die Bedeutung des Beweismittels zu berücksichtigen, sprich, ob es das einzige Beweismittel ist, oder ob für eine Verurteilung weitere Beweise zur Verfügung stehen, die rechtmässig erhoben wurden. ${ }^{110}$

106 Schmid (Fn. 86), S. 5.

107 BGE 109 Ia 244 E. 2b S. 246 = Pra 72 (1983) Nr. 27.

108 Vorne Kapitel II.1 und II.2.

109 Vgl. auch Mäder (Fn. 30) S. 162, insb. Hinweis in Fn. 77.

110 Vest/Höhener (Fn. 96), S. 99; allerdings weist Ruckstuhl zutreffend darauf hin, dass sich die entgegenstehenden Interessen immer die Waage halten. Ist der fragliche Beweis nicht ausschlaggebend, so sinkt das Interesse des Staates an seiner Verwertung in gleichem Mass wie das Interesse des Beschuldigten an seiner Unverwertbarkeit. Umgekehrt steigen die Interessen beider Parteien, wenn es sich um den einzigen Beweis handelt (Ruckstuhl Niklaus, Rechtswidrige Beweise erlaubt, Beilage zu Plädoyer 6/2006, S. 19).
71 Neben diesen Individualinteressen müssen ferner die öffentlichen Interessen berücksichtigt werden.

72 Die Rechts- und Verwertungsregeln müssen so angewandt werden, dass kein Anreiz zu Selbstjustiz bei der Beweissammlung besteht.111 Je eher eine Dashcam-Aufzeichnung zur Verwertung zugelassen wird, desto grösser ist die Gefahr, dass sich Private zu «Hilfssheriffs»112 aufschwingen und zur Selbstjustiz greifen. Dies ist gerade dann problematisch, wenn der Private weder geschädigt noch beeinträchtigt ist, sodass der Private in einem entsprechenden Verfahren an solchen Beweisen selbst kein Interesse hat, ${ }^{113}$ er also quasipolizeiliche Aufgaben wahrnimmt und die Grenzen individueller Gefahrenabwehr überschreitet. ${ }^{114}$ Deshalb sollte nach der hier vertretenen Ansicht in der Interessenabwägung berücksichtigt werden, ob der Kamerabetreiber überhaupt Verletzter der Straftat ist, ${ }^{115}$ bzw. ob er durch die Straftat gefährdet wurde.

73 Zudem bestehen gewichtige öffentliche Interessen an einem fairen Verfahren

111 Gless, in: BSK-StPO I (Fn. 45), Art. 141 Rz. 42.

112 So etwa das Amtsgericht Nienburg, Urteil v. 20.01.2015 - 4 Ds 520 Js 39473/14 (155/14), ZD 2015, 341 (343); ebenso Entscheid des Bezirksgerichts Schwyz vom 20. Oktober 2016 (SEO 2016 19), E. 1.6.3.

113 Vgl. Entscheid des Kantonsgerichts Schwyz STK 20171 vom 20. Juni 2017, E. 3b.cc.

114 Vgl. Müller (Fn. 10), S. 316 und 346 ff.; Pieth hält fest, dass dort, wo Private quasi als Behörde auftreten (etwa, wenn sie eine Bürgerwehr bilden, welche systematisch Jagd auf Drogenhändler macht), die Regeln für staatliche Beweisgewinnung gelten, selbst wenn die Initiative von Privaten ausgeht (Pieth Mark, Schweizerisches Strafprozessrecht, 3. Aufl., Basel 2016, S. 196).

115 Vgl. auch Roggenkamp Jan Dirk, DashcamAufnahmen als Beweis im Zivil- und Strafprozess, in: AnwZert ITR 10/2015 Anm. 3. 
sowie an der Justizförmigkeit des Verfahrens. ${ }^{116}$ Jeder Rückgriff der Behörden auf illegal erlangte Beweise erschüttert das Vertrauen der Öffentlichkeit in eine rechtsstaatliche Strafrechtspflege. ${ }^{117}$ Es besteht ein öffentliches Interesse daran, dass keine Straftaten begangen werden auch nicht bei Beweismittelerhebungen durch Private. ${ }^{118}$ Dies muss auch für «schlichte» Rechtsverletzungen gelten.

74 Demgegenüber bestehen öffentliche Interessen an der Aufklärung von Straftaten (Wahrheitsfindung), ${ }^{119}$ an der Effektivität der Verfolgung von strafrechtlichem Fehlverhalten (effektiver Rechtsschutz) ${ }^{120}$ sowie an der Gewährleistung der Sicherheit des öffentlichen Strassenverkehrs und damit dem Schutz von Gesundheit und Leben der Verkehrsteilnehmer bzw. dem Schutz von Grundrechten Dritter. ${ }^{121}$

75 Stellt man diese Abwägungskriterien einander gegenüber, so überwiegen die privaten und öffentlichen Interessen an

$116 \overline{\text { Entscheid des Kantonsgerichts Schwyz STK } 2017}$ 1 vom 20. Juni 2017, E. 3b.cc.

117 Gless Sabine, Beweisverbote und Fernwirkung, in: ZStrR 128/2010 (nachfolgend zit.: Gless, Beweisverbote), S. 157.

118 Schmid (Fn. 86), S. 6.

119 Entscheid des Kantonsgerichts Schwyz STK 2017 1 vom 20. Juni 2017, E. 3b.cc; Entscheid des Bezirksgerichts Schwyz vom 20. Oktober 2016 (SEO 2016 19), E. 1.6.3.

120 Entscheid des Bezirksgerichts Schwyz SEO 2016 19 vom 20. Oktober 2016, E. 1.6.3; Oberlandesgericht Stuttgart, Beschluss v. 04.05.2016 - 4 Ss 543/15, NJW 2016, 2280 (2281); Amtsgericht Nienburg, Urteil v. 20.01.2015 - 4 Ds 520 Js 39473/14 (155/14), ZD 2015, 341 (343).

121 Entscheid des Bezirksgerichts Schwyz vom 20. Oktober 2016 (SEO 2016 19), E. 1.6.3; vgl. auch Rütsche Bernhard, in: Niggli/Probst/Waldmann (Hrsg.), Basler Kommentar, Strassenverkehrsgesetz, Basel 2014, vor Art. 16-17a Rz. 4; vgl. ebenfalls Oberlandesgericht Stuttgart, Beschluss v. 04.05.2016 - 4 Ss 543/15, NJW 2016, 2280 (2281). der Unverwertbarkeit von DashcamAufzeichnungen bei einfachen Verkehrsregelverletzungen nach Art.90 Abs. 1 SVG (als Übertretungen) wohl regelmässig. Demgegenüber überwiegen die Interessen an der Verwertbarkeit von Dashcam-Aufzeichnungen bei schweren Straftaten ${ }^{122}$ tendenziell.

76 Bei groben Verkehrsregelverletzungen nach Art. 90 Abs. 2 SVG dürfte es sich um einen Grenzfall handeln: Einerseits stellen grobe Verkehrsregelverletzungen (als Vergehen) nur relativ schwerwiegende nicht aber schwere Straftaten dar, ${ }^{123}$ was tendenziell für die Unverwertbarkeit von Videoaufzeichnungen spricht, auf denen solche grobe Verkehrsregelverletzungen $\mathrm{zu}$ sehen sind. ${ }^{124}$ Andererseits setzt der objektive Tatbestand von Art. 90 Abs. 2 SVG (neben der groben Verletzung einer Verkehrsregel) voraus, dass eine ernstliche Gefahr für die Sicherheit anderer hervorgerufen oder in Kauf genommen wird. Eine ernstliche Gefahr für die Sicherheit anderer ist gemäss Bundesgericht nicht erst bei einer konkreten, sondern bereits bei einer erhöhten abstrakten Gefährdung gegeben. ${ }^{125}$ Diese konkrete bzw. zumindest

$122 \overline{\text { Zum Begriff der «schweren Straftat» vgl. vorne }}$ Rz. 67.

123 Vgl. vorne Rz. 67.

124 Vgl. etwa Uttinger (Fn. 102), Rz. 13; vgl. auch Mäder (Fn. 30), S. 167.

125 BGE 131 IV 133 E. 3.2 S. 136 m.w.H. «Ob eine konkrete, eine erhöhte abstrakte oder nur eine abstrakte Gefahr geschaffen wird, hängt von der Situation ab, in welcher die Verkehrsregelverletzung begangen wird. Wesentliches Kriterium für die Annahme einer erhöhten abstrakten Gefahr ist die Nähe der Verwirklichung. Die allgemeine Möglichkeit der Verwirklichung einer Gefahr genügt demnach nur zur Erfüllung des Tatbestands von Art. 90 Ziff. 2 SVG, wenn in Anbetracht der Umstände der Eintritt einer konkreten Gefährdung oder gar einer Verletzung nahe liegt.» 
erhöhte abstrakte Gefährdung anderer als Folge der groben Verkehrsregelverletzung spricht tendenziell für eine Verwertung solcher Aufzeichnungen.

77 In jedem Fall ist aber eine detaillierte und einzelfallbezogene Interessenabwägung anhand obiger Abwägungskriterien vorzunehmen. Dabei ist die DashcamAufzeichnung insbesondere dann nicht verwertbar, wenn bei ihrer Beschaffung ein Rechtsgut verletzt wurde, welches im konkreten Fall den Vorrang vor dem Interesse an der Durchsetzung des Strafrechts verdient. ${ }^{126}$

\section{Rechtmässige bzw. gerechtfertigte Dashcam-Aufzeichnungen}

78 Erlangen Private eigeninitiativ, sprich weder im Auftrag noch mit Unterstützung der Strafbehörden, und rechtmässig Beweismittel, sind diese grundsätzlich verwertbar. ${ }^{127}$ Wurden Dashcam-Aufzeichnungen rechtskonform, sprich insbesondere im Einklang mit dem Zivil-, dem Datenschutz- sowie dem Strafrecht, erhoben, sind diese im Strafverfahren als Beweismittel demnach zuzulassen.

79 Überdies stellt sich die Frage der Unverwertbarkeit dann überhaupt nicht, wenn das Verhalten des Privaten (auf materiell-rechtlicher Ebene) durch einen

$126 \overline{\text { Vgl. BGE } 131 \text { I } 272 \text { E. 4.1.2 S. } 279 \text { mit Hinweis auf }}$ Urteil P.1152/1987 vom 10. Dezember 1987, E. 3a, publ. in: ZBl 9o/1989, S. 420; vgl. noch Art. 150 VE-StPO.

127 Gless, in: BSK-StPO I (Fn. 45), Art. 141 Rz. 40c; Demgegenüber sprechen Riedo/Fiolka/Niggli davon, dass durch Private rechtmässige Beweise «ohne weiteres» und damit in jedem Fall verwertbar sind (Riedo/Fiolka/Niggli [Fn. 53], § 30 Rz. 1074).
Rechtfertigungsgrund gedeckt ist. ${ }^{128}$ Insbesondere wenn es dem Privaten nicht primär um die Überführung des Straftäters, sondern um die Abwehr unrechtmässiger Eingriffe geht, ist im Einzelfall zu prüfen, ob der Geschädigte aufgrund von Notwehr (Art. 15 f. StGB), Notstand (Art. $17 \mathrm{f}$. StGB) sowie nach dem Grundsatz der Wahrung berechtigter Interessen befugt gewesen ist, in dringenden Fällen sowie unter Beachtung der Verhältnismässigkeit selbst zugunsten der Strafverfolgungsbehörden Beweise zu sichern. ${ }^{129}$ Dies ist etwa dann der Fall, wenn nötigende Äusserungen bzw. Verhaltensweisen unerlaubterweise auf einem Bildoder Tonspeichergerät aufgenommen werden. ${ }^{130}$

\section{Entlastende Dashcam- Aufzeichnungen als Sonderfall}

80 Ebenfalls stellt sich die Frage, ob unverwertbare Dashcam-Aufzeichnungen auch in Bezug auf mögliche Entlastungsbeweise einem Beweisverwertungsverbot unterliegen. Denkbar ist etwa, dass sich eine rechtswidrig erhobene Dashcam-Aufnahme zu Gunsten des Beschuldigten auswirkt.

81 Vor Einführung der Strafprozessordnung waren Beweise, welche sich zu Gunsten des Beklagten auswirkten, grundsätzlich

128 Schmid/Jositsch, Handbuch StPO (Fn. 51), Rz. 802; dies., PraxKomm-StPO (Fn. 52), Art. 141 Rz. 3; Godenzi, Diss. (Fn. 52), S. 166 und 170 f.

129 Hauser Robert/Schweri Erhard/Hartmann Karl, Schweizerisches Strafprozessrecht, 6. Aufl., Basel 2005, § 60 Rz. 14; Schmid/Jositsch, Handbuch StPO (Fn. 51), Rz. 802; dies., PraxKomm-StPO (Fn. 52), Art. 141 Rz. 3.

130 Schmid/Jositsch, PraxKomm-StPO (Fn. 52), Art. 141 Rz. 3; vgl. auch Entscheid des Kantonsgerichts Wallis P1 1365 vom 24. Juni 2014, E. 3.4.1.1. 
verwertbar. ${ }^{131}$ Strittig ist, ob Beweisverbote nach der neuen Strafprozessordnung nur Belastungs- oder auch Entlastungsverbote sind. ${ }^{132}$

Die wohl überwiegende Lehre und die noch spärlich vorhandene Rechtsprechung befürworten tendenziell ein blosses Belastungsverbot.133 Begründet wird dies zunächst damit, dass eine ausdrückliche gesetzliche Grundlage fehlt. Im Begleitbericht VE-StPO heisst es denn auch explizit, dass es der Praxis überlassen bleiben könne, «inwieweit solche Beweise zugunsten der Beschuldigten oder anderer Parteien verwendet werden».134 Daneben wird angeführt, dass die Strafjustiz es sich nicht leisten könne, irgendein vorhandenes Indiz zur Unschuld des

${ }_{131}$ Entscheid des Obergerichts des Kantons Zürich UH120368 vom 24. April 2013, E. 3.2.3b.

${ }^{132}$ Gless, in: BSK-StPO I (Fn. 45), Art. 141 Rz. 113; vgl. auch Thommen Marc/Seelmann Martin, Entscheidanmerkung zum Urteil SB150061 des Obergerichts des Kantons Zürich, in: forumpoenale 5/2016, S. 260.

133 Entscheid des Kantonsgerichts Graubünden SK1 154 vom 4. Oktober 2016, E. 3b; Entscheid des Obergerichts des Kantons Zürich SB150061 vom 3. Dezember 2015, E. 3.6 und 3.7; Entscheid des Obergerichts des Kantons Zürich UH120368 vom 24. April 2013, E. 3.2.3b; Entscheid des Obergerichts des Kantons Bern BK 201262 vom 18. Juni 2012, E. 4.2; Bénédict/Treccani, in: CR-CPP (Fn. 52), Intro Art. 139-141 Rz. 18 f. und Art. 141 Rz. 32 f.; Gless, in: BSK-StPO I (Fn. 45), Art. 141 Rz. 115 f.; dies., Beweisverbote (Fn. 117), S. 160; Jositsch (Fn. 55), § 58 Rz. 280; Parein Saskia, Les preuves illégales recueillies par les particuliers sous l'empire du Code de procédure pénale suisse, in: Jusletter vom 8. Oktober 2012, Rz. 52; Riklin (Fn. 54), Art. 141 Rz. 9; Schmid/Jositsch, Handbuch StPO (Fn. 51), Rz. 792; Thommen/Seelmann (Fn. 132), S. 259 und 261; a.M. Donatsch Andreas/Cavegn Claudine, Ausgewählte Fragen zum Beweisrecht nach der schweizerischen Strafprozessordnung, in: ZStrR 126/2008, S. 166 f.; Wohlers, in: StPO-Komm. (Fn. 52), Art. 141 Rz. 12 und 19.

134 Eidgenössisches Justiz- und Polizeidepartement, Begleitbericht zum Vorentwurf für eine Schweizerische Strafprozessordnung, Bern 2001 (Begleitbericht VE-StPO), S. 108.
Beschuldigten zurückzuweisen. ${ }^{135}$ Auch rein faktische Probleme, welche die Gegenseite anbringt, überzeugten nicht. Eine praktikable Lösung müsse etwa auch für Konstellationen gefunden werden, in welchen ein Verwertungsverbot nicht bei allen Beschuldigten greift, sondern nur bei Einzelnen. ${ }^{136}$ Zudem sollen die Strafbehörden für Verfahrensrechtsverletzungen bestraft und von künftigen Verletzungen abgehalten werden, indem rechtswidrig erhobene Beweise nicht gegen den Beschuldigten verwertet werden dürfen, was dem fairen Verfahren dient. ${ }^{137}$ Überdies ist Häring der Auffassung, dass der Gedanke, einen offensichtlich Unschuldigen $\mathrm{zu}$ verurteilen, weil entlastende Beweise nicht verwertet werden dürfen, unerträglicher erscheint als der Gedanke, einen offensichtlich Schuldigen mangels verwertbarer Beweise freizusprechen. ${ }^{138}$

83 Schliesslich hält das Kantonsgericht Graubünden fest, dass dem Grundsatz, wonach eine Verurteilung nur erfolgen darf, wenn der Beschuldigte sich in objektiver und subjektiver Hinsicht nach-

$135 \overline{\text { Walder Hans, Rechtswidrig erlangte Beweismittel }}$ im Strafprozess, in: ZStrR 82/1966, S. 50.

${ }_{136}$ Vgl. Art. 147 Abs. 4 StPO; Gless, in: BSK-StPO I (Fn. 45), Art. 141 Rz. 115; So können die praktischen Probleme entweder durch eine Verfahrenstrennung oder eine doppelte Aktenführung gelöst werden (Gless, in: BSK-StPO I [Fn. 45], Art. 141 Rz. 116), sofern es dem Gericht nicht ohnehin zuzumuten ist, unverwertbare Beweise in seiner Entscheidung nicht zu berücksichtigen, obwohl sie nach wie vor Bestandteil der Akten bilden (Entscheid des Obergerichts des Kantons Zürich UH120368 vom 24. April 2013, E. 3.2.3c).

137 Thommen/Seelmann (Fn. 132), S. 261; Thommen Marc/Samadi Mojan, The Bigger the Crime, the Smaller the Chance of a Fair Trial?, European Journal of Crime, Criminal Law and Criminal Justice 2016, S. $81 \mathrm{ff}$.

${ }^{138}$ Häring (Fn. 52), S. 236; vgl. auch Entscheid des Obergerichts des Kantons Bern BK 201262 vom 18. Juni 2012, E. 4.2. 
weislich tatsächlich schuldig gemacht hat, grundlegende Bedeutung zukommt. Diese Wertung finde sich etwa im fundamentalen Grundsatz «in dubio pro reo». 139

84 Der wohl überwiegenden Lehrmeinung sowie der spärlichen Rechtsprechung zu Entlastungsbeweisen unter der neuen StPO ist zu folgen, weshalb den Beschuldigten entlastende Dashcam-Aufzeichnungen zu dessen Gunsten zu verwerten sind. ${ }^{140}$ Zum einen ist es stossend, eine offensichtlich unschuldige Person zu verurteilen. Zum anderen stehen gegen den fehlbaren Dashcam-Verwender zivilund u.U. strafrechtliche Rechtsbehelfe zur Verfügung, um diesen für den unzulässigen Dashcam-Einsatz zu sanktionieren.

85 Wo eine illegale Dashcam-Aufzeichnung einen Automobilisten entlastet, einen anderen hingegen belastet, ist die unverwertbare Aufzeichnung zugunsten des Automobilisten, welcher dadurch entlastet wird, zu verwerten. Allerdings müssen die Interessen des Mitbeschuldigten, nicht gestützt auf einen illegalen Beweis verurteilt zu werden, berücksichtigt werden, weshalb der entsprechende Beweis dann nicht gegen den Mitangeschuldigten verwertet werden könnte. ${ }^{141}$

$139 \overline{\text { Entscheid des Kantonsgerichts Graubünden SK} 1 ~}$ 154 vom 4. Oktober 2016, E. 3 b.

140 Obwohl das Argument, wonach dem Beschuldigten ein begünstigender Beweis nicht entzogen werden soll, weil die Strafbehörden ihn rechtsfehlerhaft erlangt haben, im Zusammenhang mit Dashcam-Aufzeichnungen nicht greift, da diese ja gerade ohne Mitwirkung der Strafbehörden erstellt wurden.

$141 \mathrm{Vgl}$. Bénédict/Treccani, in: CR-CPP (Fn. 52), Intro Art. 139-141 Rz. 18 f. und Art. 141 Rz. 32 f.; vgl. auch Entscheid des Obergerichts des Kantons Bern vom 18. Juni 2012 (BK 2012 62), E. 4.2; im Ergebnis entspricht dies auch dem Entscheid des

\section{Prüfschema zur Verwertbarkeit privater Dashcam-Aufzeichnungen}

86 Das folgende Prüfschema dient als Überblick, wie vorzugehen ist, wenn die Frage nach der Verwertbarkeit einer DashcamAufzeichnung im Raum steht.
Obergerichts des Kantons Zürich UH120368 vom 24. April 2013, insb. E. 3.2.2b, E. 3.2.3c sowie E. 3.3 . 


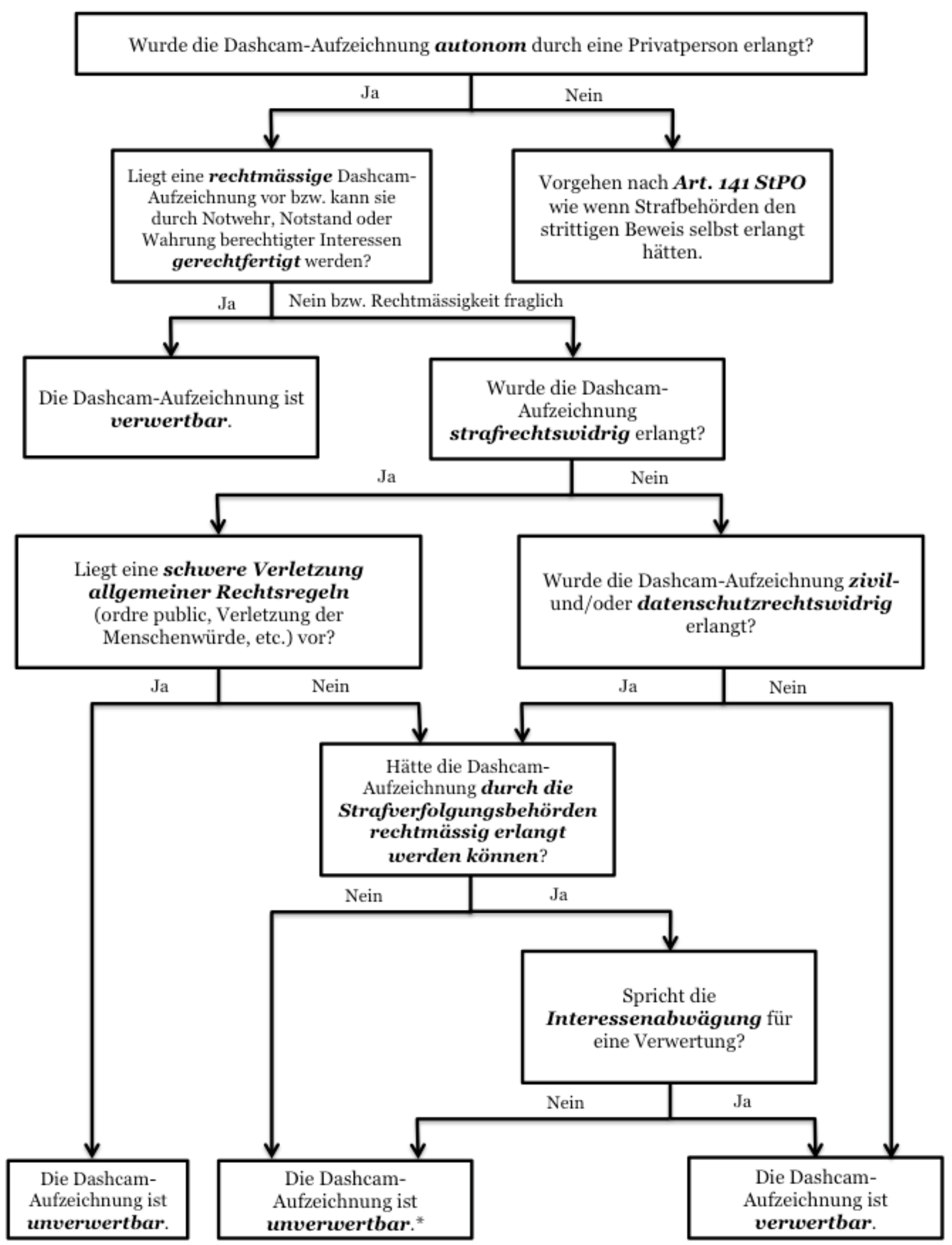

*Den Beschuldigten entlastende Dashcam-Aufzeichnungen sind allerdings zu dessen Gunsten zu verwerten. 


\section{Fazit}

87 Wie sich aus dem vorliegenden Beitrag ergibt, bestehen bei der Frage nach der Verwertbarkeit von Dashcam-Aufzeichnungen nach wie vor erhebliche Rechtsunsicherheiten. Auch die ersten ergangenen Gerichtsentscheide zur Verwertbarkeit solcher Aufzeichnungen konnten nur beschränkt Licht ins Dunkel bringen.

88 Obwohl Dashcam-Aufzeichnungen regelmässig «lediglich» zivil- und datenschutzrechtswidrig erlangt werden, sind solche Aufzeichnungen - gleich wie strafrechtswidrig erlangte Beweise - nur verwertbar, wenn sie einerseits von den Strafverfolgungsbehörden rechtmässig hätten erlangt werden können und andererseits eine Interessenabwägung für ihre Verwertung spricht.

89 Die hypothetisch rechtmässige Beweiserlangung ist dann zu bejahen, wenn der Private die Videoaufnahme aufgrund konkreter Verdachtsmomente erstellt, sowie bei anlasslosen privaten Aufzeichnungen, wenn die Polizei nach ersten Verdachtsmomenten noch eine Kamera hätte einschalten können.

90 In einer Vielzahl der Fälle dürfte der zweiten Voraussetzung, der Interessenabwägung, bei der Frage der Verwertbarkeit von Dashcam-Aufzeichnungen entscheidende Bedeutung zukommen. Bei einfachen Verkehrsregelverletzungen führt diese Abwägung regelmässig zur Unverwertbarkeit der Dashcam-Aufzeichnungen, bei schweren Straftaten im obigen Sinne tendenziell zur Verwertbarkeit. In jedem Fall ist aber eine einzelfallbezogene Interessenabwägung anhand der erwähnten Abwägungskriterien vorzunehmen.
91 Aufgrund dieser Einzelfallbetrachtung kann und wird es in naher Zukunft wohl keine pauschale Erlaubnis oder ein pauschales Verbot von Dashcam-Aufzeichnungen geben. Es liegt somit an den Gerichten, durch nachvollziehbare und konsistente Entscheidungen Rechtssicherheit zu schaffen. 